\title{
The phase-integral method and black hole normal modes
}

\author{
N Andersson $\dagger, M$ E Araújo $\$ \S \|$ and B F Schutzף \\ Department of Physics and Astronomy, University of Wales College of Cardiff, \\ Cardiff CF2 3YB, UK
}

Received 4 September 1992

\begin{abstract}
The phase-integral method has proved to be a powerful tool for studying the quasinormal modes of black holes. A generalization of the wKB methods of quantum mechanics, its treatment of the complex coordinate plane brings a number of important simplifications and potentially powerful computational aids to bear on the problem of computing eigenfrequencies with large imaginary parts. It holds great promise of further applications to related problems, such as the quasinormal modes of relativistic stars. However, in some respects the method is incomplete, particularly in its assessment of error bounds. This paper makes available to researchers in the field of relativity a simple and self-contained introduction to the fundamental concepts of the phase-integral method, in which we also point out areas that seem to need further development. As an example of the use of the method, we derive the two-transition-point phase-integral formula for quasinormal modes of the Schwarzschild black hole, which is an accurate asymptotic approximation for the first modes. The present paper provides the foundation for related papers in which we use the method to develop accurate asymptotic expressions for highly damped modes.
\end{abstract}

PACS numbers: 0270, 0420, 0430, 9760L

\section{Introduction}

\subsection{Background}

The quasinormal modes of a radiating system in general relativity are solutions of the perturbation equations that satisfy the 'causal' boundary condition that the waves be outgoing at infinity. In addition, if the system is a black hole, one requires as well the causal condition that the waves be ingoing at the horizon. These conditions generally lead to a set of discrete complex eigenfrequencies $\left\{\sigma_{j}\right\}$, whose imaginary parts usually have the appropriate sign to provide the damping expected for such boundary conditions. However, it is of course possible that a particular mode could represent an instability, and then the eigenfrequency's imaginary part will have the other sign. Rather unexpectedly, it has been found that these boundary conditions

† E-mail address: andersson@cardiff.ac.uk

$\ddagger$ E-mail address: araujo@taff.cardiff.ac.uk

§ Permanent address: Departamento de Matemática, Universidade de Brasília, Caixa Postal 04670, 70.919-000 Brasília DF, Brazil.

|| Permanent e-mail address: marcelo@br.unb.bitnet

If E-mail address: schutz@cardiff.ac.uk 
lead to sequences of eigenfrequencies with ever-increasing imaginary parts, i.e. which damp faster and faster. This was discovered for black holes by Leaver [1]. For stars it was predicted and verified by Kokkotas and Schutz [2,3] and also by Kojima [4]. In general, we know rather little about the properties of the quasinormal modes of most systems; only the Schwarzschild case has been studied in much detail.

For a review of the fundamental theory of black hole perturbations, including references to early work, see [5]. The quasinormal modes may be an important source of gravitational waves emitted in any dynamical process involving a black hole. Investigations of slightly perturbed black holes show that the quasinormal-mode oscillations will dominate the emitted radiation at late times [6]. For non-spherical gravitational collapse there are situations where the bulk of the radiation is emitted via the quasinormal modes $[7,8]$. With the new generation of gravitational-wave detectors that is being developed $[9,10]$, it is conceivable that this radiation will be detected. Because the quasinormal-mode frequencies are characteristic of the black hole and depend only on its mass, charge and angular momentum, an identification of these frequencies in a detected gravitational-wave spectrum would in principle enable the positive identification of a black hole and the determination of all its parameters. Hence, there is a need for reliable theoretical predictions and an increased knowledge of these processes.

We know much less about the quasinormal modes of stars. For a review of stellar quasinormal-mode theory before the discovery of the very strongly damped modes, but including a discussion of the rather surprising result that coupling to gravitational radiation can induce instabilities, see Friedman [11]. The quasinormal modes of stars will be equally observable by gravitational-wave detectors, but computational complexities have so far prevented a thorough theoretical study of their properties, even for non-rotating stars.

The basic difficulty of calculating quasinormal-mode frequencies that have significant imaginary parts is well known. The main point is that it is difficult to resolve numerically the two independent solutions of the differential equation near infinity, because for a damped wave the outgoing-wave solution grows exponentially while the ingoing one dies exponentially. For a black hole, this problem also occurs at the event horizon. A number of methods have been applied to the study of Schwarzschild quasinormal modes to get around this problem. The original discovery of the very strongly damped modes by Leaver [1] resulted from his solving the recurrence relation for a series solution of the wave equation by transforming the relation into a continued fraction. This discovery in turn stimulated a number of alternative approaches to try to find more transparent methods of finding modes, and to check Leaver's numerical results. Among these is the elegant treatment of Nollert and Schmidt [12] in which the quasinormal mode frequencies are defined as poles of the Green function for the Laplace-transformed wave equation.

Approximate analytic methods have also been applied to the problem; see for example Ferrari and Mashhoon [13] and references therein. The application of WKB-type methods in the complex coordinate plane was first suggested by Guinn et al [14], who obtained good agreement with Leaver for the first few modes but found significant differences when the damping increased. Slightly better agreement for highly damped modes was found by Fröman et al [15] in the first application of the phase-integral method, which is a generalization of the WKB method. In a direct application of the formulae developed in this article the present authors [16] have obtained results in agreement with those of Leaver and Nollert and Schmidt. 
Moreover, Nollert $[17,18]$ applied asymptotic methods to the same recurrence relations used by Leaver, and found the asymptotic form of the eigenfrequencies for very high index numbers (much larger than Leaver had examined). The full power of the phase-integral method has become apparent with Andersson and Linnæus' [19] verification of Nollert's asymptotic result [20].

The importance of the phase-integral method is its wide potential applicability. Methods that use recurrence relations may need relations of a particular form, and may not generalize to other systems of interest. The phase-integral method uses essentially only one feature of the problem: that there is an analytic wave equation which can be continued into the complex coordinate plane when the eigenfrequency is complex. The method therefore offers results not only for black holes but for the treatment of the exterior solution to the wave equation for spherical and slowlyrotating stars. As an analytic method, it also offers the possibility of making the numerical results more intuitive. Moreover, as we note below, it provides a way around the numerical problems that occur near infinity: it shows one how to do the problem even by numerical integration, without running the risk of losing one of the independent solutions in the numerical noise [21].

\subsection{Mathematical formulation}

For a Schwarzschild black hole, and for the exterior of a spherical star, the fundamental perturbation equations can be reduced to a single ordinary differential equation by separating out the dependence on time and angles. Assuming that the time dependence is $\exp [-\mathrm{i}(\sigma / M) t]$, and fixing the spherical-harmonic index $\ell$, we obtain a time-independent Schrödinger-like differential equation for a wave variable $\Psi$

$$
\frac{\mathrm{d}^{2} \Psi}{\mathrm{d} r^{2}}+R_{\ell}(r) \Psi=0
$$

where $\Psi$ is related to the solution, $\Psi_{\mathrm{RW}}$, to the Regge-Wheeler equation by

$$
\Psi=\sqrt{1-2 / r} \Psi_{\mathrm{RW}}
$$

From $\Psi_{\mathrm{RW}}$ all components of the perturbed metric tensor can be reconstructed [5].

If we scale the usual Schwarzschild coordinate $r$ by the mass, $M$, of the black hole, then $R_{\ell}$ takes the form

$$
R_{\ell}(r)=\frac{r^{2}}{(r-2)^{2}}\left[\sigma^{2}-V_{\ell}(r)+\frac{2}{r^{3}}-\frac{3}{r^{4}}\right]
$$

where the Regge-Wheeler potential $V_{\ell}(r)$ [22] is given by

$$
V_{\ell}(r)=\left(1-\frac{2}{r}\right)\left[\frac{\ell(\ell+1)}{r^{2}}-\frac{6}{r^{3}}\right] \text {. }
$$

Note that the function $R_{\ell}$ is more complicated than the Regge-Wheeler potential because of the transformation of the dependent variable that has been applied to the usual Regge-Wheeler equation in order to put it into the standard Schrödinger form with the Schwarzschild coordinate $r$ as the independent variable. (Relativists may be 
more familiar with a different transformation in which $r$ is changed to the 'tortoise' coordinate $r_{*}$. We avoid that transformation here because it introduces logarithmic singularities.) Note also that $R_{\ell}$ contains the eigenfrequency, so when one iterates over a set of frequencies to find the right one, the function $R_{\ell}$ changes. Moreover, it may be worth pointing out that this means that the analysis described below will be equally applicable to problems where the potential $V_{l}$ is explicitly dependent on the frequency. This is the case for perturbations of Kerr black holes.

One obtains the same complex frequencies from (4) as with the alternative potential derived by Zerilli [23], as is shown in [24] and [25]. In the present work we will only consider the potential given by (4) because it has a simpler functional form.

It was shown by Regge and Wheeler [22] (see Chandrasekhar [5]) that the energy integral, together with the given boundary conditions, leads to damped quasinormal modes: there are no instabilities. Therefore, with our choice of time factor we must have $\operatorname{Im} \sigma<0$.

The purpose of this paper is to discuss the application of the phase-integral method to the quasinormal-mode problem. In the phase-integral method, developed in a book [26] and several papers by Fröman and Fröman, a local solution to (1) is given by a symmetric phase-integral approximation. To the lowest order this approximation is equivalent to the first-order WKB approximation. Taken to higher orders $[27,28]$ the phase-integral approximation has, in general, the same formal accuracy as the WKB approximation of higher orders. It is easier to establish error bounds in the phase-integral method, although we shall see that these bounds still leave something to be desired. Moreover, the Wronskian of any two exact linearly independent solutions to (1) must be constant. This property is preserved in the phase-integral approximation, but not for the higher-order WKB solutions. This constancy of the Wronskian at any order of approximation allows for a systematic use of higher orders in continuing an approximate solution in the complex coordinate plane. However, the notation used has changed through the years, thus obscuring the reading of many of the original papers. Therefore, we will review the phase-integral method in this paper. All the formulae and ideas relevant to a phase-integral analysis of the quasinormal-mode problem will be discussed.

For a discussion of the much wider context in which the method finds applications, and for further references, we suggest a recent article [29]. The use of $F$-matrices (see below) to solve connection problems is extensively discussed in [30]. Naturally there are alternatives to the method devised by Fröman and Fröman. A nice review of semiclassical methods was written by Berry and Mount [31]. An extensive description of the history of phase-integral methods can be found in Heading's book [32]. Although these references are 20 and 30 years old, respectively, they are still worth reading. For a comprehensive textbook description of the WKB approximation we refer the reader to $[33,34]$.

\section{The phase-integral method}

\subsection{Fundamental concepts}

In the phase-integral method the approximate solution to (1) is continued into the complex $r$-coordinate plane. Consequently all functions of $r$ considered hereafter are complex-valued analytic functions. 
The most general transformation of the dependent and independent variables that preserves the reduced form of (1) is given in terms of an arbitrary function $q$, a redefined wave variable $\phi$, and a new independent variable $w$. We define [26]

$$
\Psi(r)=q^{-1 / 2}(r) \phi(r)
$$

and

$$
w(r)=\int^{r} q(\xi) \mathrm{d} \xi .
$$

Equation (1) transforms to the convenient form

$$
\frac{\mathrm{d}^{2} \phi}{\mathrm{d} w^{2}}+(1+\epsilon) \phi=0
$$

where

$$
\epsilon=\frac{R_{\ell}-q^{2}}{q^{2}}+q^{-3 / 2} \frac{\mathrm{d}^{2}}{\mathrm{~d} r^{2}}\left(q^{-1 / 2}\right)
$$

should be thought of as a functional $\epsilon[q]$. We note in passing that, by using an exact relation obtained by Dammert [35],

$q^{-3 / 2} \frac{\mathrm{d}^{2}}{\mathrm{~d} r^{2}}\left(q^{-1 / 2}\right)=-\left(\frac{1}{2 m} q^{-m-1} \frac{\mathrm{d}}{\mathrm{d} r} q^{m}\right)^{2}-\frac{\mathrm{d}}{\mathrm{d} w}\left(\frac{1}{2 m} q^{-m-1} \frac{\mathrm{d}}{\mathrm{d} r} q^{m}\right)$

where $m$ is an arbitrary real number $(\neq 0)$, one can write the definition of $\epsilon$ in various different and useful ways.

Let us now assume that the function $q(r)$ is somehow chosen to be an exact solution to (8) with $\epsilon=0$ in some region of the complex $r$-plane. Then the two functions

$$
\phi_{ \pm}(r)=\mathrm{e}^{ \pm \mathrm{i} w(r)}
$$

are linearly independent exact solutions to (7). The general solution to (1) is then given by the linear combination

$$
\Psi(r)=a_{1} f_{1}(r)+a_{2} f_{2}(r)
$$

where

$$
f_{1}(r)=q^{-1 / 2}(r) \mathrm{e}^{\mathrm{i} w(r)} \quad f_{2}(r)=q^{-1 / 2}(r) \mathrm{e}^{-\mathrm{i} w(r)} .
$$

Of course, if the function $R_{\ell}(r)$ has a complicated functional form it is generally not possible to find an exact solution to $\epsilon=0$. However, it may be possible to find an approximation to $q(r)$ in such a way that $|\epsilon|$ is small compared to unity. Then (12) still gives approximate solutions to the differential equation (1). This will be our approach below.

The usual WKB approximation is obtained by setting $q^{2} \approx R_{\ell}$. Provided that the second term on the right-hand side of $(8)$ is sufficiently small, this approximation is accurate. This requirement corresponds, roughly speaking, to saying that $R_{\ell}(r)$ is a slowly varying function of the complex coordinate $r$-see for example [33]. 


\subsection{The phase-integral approximation}

As pointed out by Fröman and Fröman (see [29] for references) it is possible to get close to an exact solution of $\epsilon=0$ by refining the function $q(r)$ from a first guess $Q(r)$ through a series of approximations. In the $(2 N+1)$ th order of approximation the function $q(r)$ is given by

$$
q(r) \approx Q(r) \sum_{n=0}^{N} Y_{2 n}
$$

with $Y_{0}=1$. If we let $\epsilon_{0}=\epsilon[Q]$, then the first $Y_{2 n}$ are

$$
\begin{aligned}
& Y_{2}=\frac{1}{2} \epsilon_{0} \quad Y_{4}=-\frac{1}{8}\left(\epsilon_{0}{ }^{2}+\frac{\mathrm{d}^{2} \epsilon_{0}}{\mathrm{~d} \zeta^{2}}\right) \\
& Y_{6}=\frac{1}{32}\left[2 \epsilon_{0}^{3}+6 \epsilon_{0} \frac{\mathrm{d}^{2} \epsilon_{0}}{\mathrm{~d} \zeta^{2}}+5\left(\frac{\mathrm{d} \epsilon_{0}}{\mathrm{~d} \zeta}\right)^{2}+\frac{\mathrm{d}^{4} \epsilon_{0}}{\mathrm{~d} \zeta^{4}}\right] \\
& Y_{8}=-\frac{1}{128}\left[5 \epsilon_{0}^{4}+30 \epsilon_{0}^{2} \frac{\mathrm{d}^{2} \epsilon_{0}}{\mathrm{~d} \zeta^{2}}+50 \epsilon_{0}\left(\frac{\mathrm{d} \epsilon_{0}}{\mathrm{~d} \zeta}\right)^{2}+10 \epsilon_{0} \frac{\mathrm{d}^{4} \epsilon_{0}}{\mathrm{~d} \zeta^{4}}+28 \frac{\mathrm{d} \epsilon_{0}}{\mathrm{~d} \zeta} \frac{\mathrm{d}^{3} \epsilon_{0}}{\mathrm{~d} \zeta^{3}}+\frac{\mathrm{d}^{6} \epsilon_{0}}{\mathrm{~d} \zeta^{6}}\right]
\end{aligned}
$$

where $\zeta$ is defined by

$$
\zeta=\int^{r} Q(\xi) \mathrm{d} \xi .
$$

If the value of $\epsilon_{0}$ is small (compared to unity) in the region of the coordinate plane under consideration, the approximation (13) is locally very accurate. The expressions for $Y_{2 n}$ are obtained by demanding that $\epsilon[q]$ must attain a minimum value at each order of approximation. Alternatively, $Y_{2 n}$ can be obtained from the well known WKB recurrence relations [27]. It is customary to describe the higher-order phase-integral approximations in terms of odd orders to retain the correspondence with the WKB approximation.

We can always choose constants in such a way that the linear combination (11) coincides with a desired exact solution to (1) at a certain point $r_{0}$. An important question is how far the approximation can be extended away from this point without becoming inaccurate. A measure of the accumulated error made in continuing a phase-integral solution from the point $r_{0}$ to $r$ along a path $\Lambda$ is given by [26]

$$
\mu_{\Lambda}\left(r, r_{0}\right)=\int_{\Lambda}|\epsilon(w) \mathrm{d} w|=\int_{\Lambda}|\epsilon(\xi) q(\xi) \mathrm{d} \xi| .
$$

This clearly is a measure of how small $\epsilon$ is in the domain of the approximation. One expects $\mu\left(r, r_{0}\right)$ to be small if $\Lambda$ runs well away from all zeros and poles of $Q^{2}(r)$, and $R(r)$ is slowly varying in the sense described above. However, our own calculations suggest that this measure generally overestimates the size of the errors, and we feel that more sensitive error bounds need to be found. 


\subsection{Treatment of the solution in the complex plane}

2.3.1. Continuation formulae. An exact solution of (7) may be represented by

$$
\phi=a_{1}(w) \mathrm{e}^{\mathrm{i} w}+a_{2}(w) \mathrm{e}^{-\mathrm{i} w}
$$

where $a_{1}$ and $a_{2}$ are expected to be slowly varying if $\epsilon$ is small. We wish to transform the second-order ordinary differential equation ( 7 ) into a coupled system of first-order ordinary differential equations for the coefficients $a_{i}$. We need to impose a further restriction on the $a_{i}$ in order to make them well defined, and we choose the standard constraint

$$
\frac{\mathrm{d} \phi}{\mathrm{d} w}=\mathrm{i} a_{1}(w) \mathrm{e}^{\mathrm{i} w}-\mathrm{i} a_{2}(w) \mathrm{e}^{-\mathrm{i} w}
$$

which is what the derivative of (17) would be if the $a_{i}$ were constant. Substituting into $(7)$ gives

$\frac{\mathrm{d} a_{1}}{\mathrm{~d} w}=\frac{1}{2} \mathrm{i} \epsilon\left[a_{1}+a_{2} \exp (-2 \mathrm{i} w)\right] \quad \frac{\mathrm{d} a_{2}}{\mathrm{~d} w}=-\frac{1}{2} \mathrm{i} \epsilon\left[a_{2}+a_{1} \exp (2 \mathrm{i} w)\right]$.

This system of differential equations can be rewritten as [26]

$$
\frac{1}{a_{1}} \frac{\mathrm{d} a_{1}}{\mathrm{~d} w}=\frac{1}{2} \mathrm{i} \epsilon\left[1+\frac{a_{2} \exp (-\mathrm{i} w)}{a_{1} \exp (\mathrm{i} w)}\right] \quad \frac{1}{a_{2}} \frac{\mathrm{d} a_{2}}{\mathrm{~d} w}=-\frac{1}{2} \mathrm{i} \epsilon\left[1+\frac{a_{1} \exp (\mathrm{i} w)}{a_{2} \exp (-\mathrm{i} w)}\right] \text {. }
$$

In the region where the two terms in (17) are of the same order of magnitude (i.e. $\left.\left|\mathrm{e}^{\mathrm{i} w}\right|=\mathrm{O}(1)\right)$, it follows from (20) that both $a_{1}$ and $a_{2}$ are approximately constant. On the other hand, in a region where one of the terms in (17) is much larger than the other, the coefficient of the dominant term remains approximately constant whereas the coefficient of the subdominant term can change substantially. Therefore, in general, different linear combinations of $f_{1}$ and $f_{2}$ have to be used to approximate the solution $\Psi$ to (1) in different regions of the complex plane.

There exist some guides as to where it is possible to continue a solution without its character changing radically. It helps to write the solution of the system (19) formally as

$$
a_{i}(w)=F_{i j}\left(w, w_{0}\right) a_{j}\left(w_{0}\right)
$$

where $w_{0} \equiv w\left(r_{0}\right)$, and $F$ is a two-by-two matrix. From (19) it follows that

$$
\operatorname{det} F\left(r, r_{0}\right)=1 \text {. }
$$

This requirement makes the inversion of (21) trivial. Moreover, the elements of the matrix $F$ (sometimes refered to as Stokes constants) were obtained as convergent series by Fröman and Fröman [26]. They obtained estimates of those series based on the assumptions that $r$ and $r_{0}\left(w\right.$ and $\left.w_{0}\right)$ can be connected by a path $\Lambda$ in the complex $r$-plane ( $w$-plane) along which $\left|\mathrm{e}^{\mathrm{i} w(r)}\right|$ increases monotonically from $r_{0}$ to $r$ and $\mu\left(r, r_{0}\right)$ is small. These estimates can be written

$$
\begin{aligned}
& \left|F_{11}\left(r, r_{0}\right)-1\right| \leqslant\left[e^{\mu}-1\right] \quad\left|F_{12}\left(r, r_{0}\right)\right| \leqslant \frac{1}{2}\left[\mathrm{e}^{\mu}-1\right]\left|\mathrm{e}^{-2 \mathrm{j} w\left(r_{0}\right)}\right| \\
& \left|F_{21}\left(r, r_{0}\right)\right| \leqslant \frac{1}{2}\left[\mathrm{e}^{\mu}-1\right]\left|\mathrm{e}^{2 \mathrm{i} w(r)}\right| \\
& \left|F_{22}\left(r, r_{0}\right)-1\right| \leqslant \frac{1}{2} \mu+\left[\mathrm{e}^{\mu}-1-\mu\right]\left|\mathrm{e}^{\left.2 \mathrm{i} \mid w(r)-w\left(r_{0}\right)\right]}\right| .
\end{aligned}
$$

In effect, $F$-matrices allow one to continue a given solution to (1) in the complex coordinate plane. In the following section we present and discuss some of the $F$-matrices that connect points in a region of the complex plane where only one transition point is present. 
2.3.2. Transition points: Stokes and anti-Stokes lines. Central to our treatment of the differential equation (1) in the complex coordinate plane is the identification of the transition points and the so-called Stokes and anti-Stokes linest.

The transition points are zeros of the function $Q^{2}(r)$. In a standard WKB analysis (where $Q^{2}=R_{\ell}$ ), these points are usually called the 'turning points' of the classical solution (especially when they are situated on the real coordinate axis). At a transition point, the phase-integral approximation (13) breaks down. In fact, in the first-order approximation $q(r)$ vanishes at the transition point and thus the transformation (5) becomes singular. Hence, the form (12) is not a good representation of the exact solution locally. Furthermore, since the error function $\epsilon[Q]$ has a pole at such a point, the higher-order expressions for $q(r)$ will be singular (and the expansion (13) clearly divergent). Therefore the phase-integral approximation must not be used in the vicinity of such points.

It is important to understand that transition points are usually simple first-order zeros of the function $Q^{2}(r)$, i.e. that the function $Q(r)$ has a branch cut emanating from the transition point. This is well known in the WKB approximation, where $Q^{2}=R_{\ell}$ is a simple analytic function. It then follows that in a small neighbourhood of such a transition point the function $R_{\ell}(r)$ can be approximated by $q_{1}\left(r-r_{0}\right)$ for a complex constant $q_{1}$ and (1) is approximately the Airy differential equation. The equation $\operatorname{Im}\left[q_{1}\left(r-r_{0}\right)\right]^{1 / 2} d\left(r-r_{0}\right)=0$ defines curves emanating from a transition point $r_{0}$. Solutions to this have a three-fold rotational symmetry about $r_{0}$ : if one solution has the form $r(\lambda)=r_{0}+c \lambda$ for some complex constant $c$ and a real parameter $\lambda$, then there will also be solutions for $c e^{2 \pi i / 3}$ and $c e^{-2 \pi i / 3}$. Therefore three such lines emanate, pairwise separated by an angle of $2 \pi / 3$ from the transition point; see figure 1. On these lines, which are called the anti-Stokes lines, the two approximate solutions (12) to our differential equation are of the same order of magnitude. The anti-Stokes lines separate different regions of exponential dominancy, i.e. they are boundaries of regions in the complex coordinate plane where one of our approximate solutions $(12)$ is dominant and the other subdominant. Likewise, three so-called Stokes lines, defined by $\operatorname{Re}\left[q_{1}\left(r-r_{0}\right)\right]^{1 / 2} d\left(r-r_{0}\right)=0$, emanate from each transition point. On these lines $\left|\mathrm{e}^{\mathrm{i} w}\right|$ attains either a maximum or a minimum.

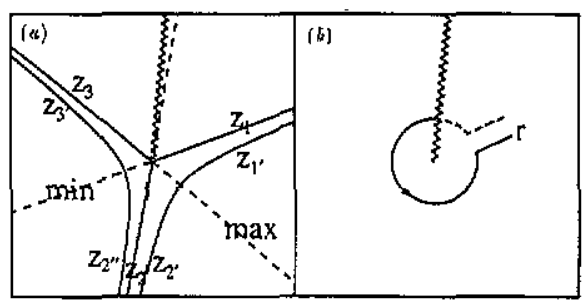

Figure 1. (a) Pattern of Stokes (broken) and anti-Stokes (full) lines in the region close to a single well separated zero of $Q^{2}(r)$. Cuts are represented by the zigzagged lines. The labels $\max$ and $\min$ on the Stokes separatrices denote maxima and minima of the function $|\exp (\mathrm{i} w)|$ respectively. $(b)$ Contour of integration used to evaluate $w$ on the Riemann surface. It is immaterial whether we circumvent the transition point in the positive or negative sense.

† A detailed description of the calculation of Stokes and anti-Stokes lines has been given by Araújo et al [36]. 
In the phase-integral method the transition points do not in general coincide with the zeros of $R_{\ell}$. In the phase-integral context Stokes (resp. anti-Stokes) lines are defined as curves along which $\mathrm{d} w$, or equivalently $Q \mathrm{~d} r$, is purely imaginary (resp. real). In other words, Stokes (resp. anti-Stokes) lines are the integral curves of the autonomous system of differential equations $\operatorname{Re}(Q \mathrm{~d} r)=0$ (resp. $\operatorname{Im}(Q \mathrm{~d} r)=0$ ). The zeros of $Q^{2}(r)$ (the transition points) are the fixed points of the system. At almost all points of the complex plane, one can find a unique Stokes and antiStokes line passing through that point, since the autonomous system of equations defining them needs only the starting point as initial data. Since a transition point has only discrete Stokes and anti-Stokes lines emerging from it, most such lines in the neighbourhood of the point avoid it.

Although the zeros of $Q^{2}(r)$ and of $R_{\ell}(r)$ generally do not coincide, in most cases one would expect them to be 'close'. That is, the distance between the zeros is such that they both lie inside the region where the phase-integral approximation deteriorates. In this case, there is an annulus surrounding both zeros within which solutions of the phase-integral equations resemble Airy functions.

Strictly speaking, the terms Stokes and anti-Stokes lines should be used to refer only to curves emanating from a transition point. In order to make a distinction, we will refer to the six lines emanating from a transition point as Stokes and antiStokes separatrices. A comparison with the theory of autonomous systems makes this nomenclature sensible. Furthermore, as mentioned above, the anti-Stokes lines emanating from a transition point separate different regions where one of the solutions (12) is exponentially dominant and subdominant, respectively.

The importance of these lines is that the solutions (10) behave as travelling waves along anti-Stokes lines. From the system (20) it is clear that the character of the solution does not alter qualitatively along an anti-Stokes line, provided of course that $\epsilon$ remains small. In fact, the changes in the qualitative behaviour of the solution that, as we noted above, can take place in the complex plane, actually take place as one moves across a Stokes separatrix [31].

In the section on the Schwarzschild problem, we will show that by placing the boundary conditions determining a quasinormal mode on anti-Stokes separatrices one obtains the correct boundary conditions at infinity and the horizon. The anti-Stokes separatrices therefore hold the key to avoiding the numerical problems of damped travelling-wave solutions near infinity: they allow one to pick out, analytically or numerically, the desired solution.

In the first-order phase-integral approximation it is customary to choose the constant lower limit of integration in (6) to be a zero of $Q^{2}(r)$. However, this is not the case when one uses higher-order phase-integral approximations because the integral is divergent at these points. In this case one replaces the definition of $w(r)$ by

$$
w(r)=\frac{1}{2} \int_{\Gamma(r)} q(\xi) \mathrm{d} \xi
$$

where $\Gamma(r)$ is a path of integration that starts at a point corresponding to $r$ on the adjacent Riemann sheet, encircles the chosen zero $t_{0}$ of $Q^{2}(r)$, and ends up at $r$. In higher orders of approximation the function $q(r)$ has several zeros in the vicinity of a zero of $Q^{2}(r)$. These 'additional' zeros, which are not branch points of $Q(r)$, must be encircled in the integration defining $w(r)$ [37]. 
2.3.3. F-matrices for a well separated transition point. As we have noted above, the phase-integral functions $f_{1}$ and $f_{2}$ are singular at transition points and the approximation breaks down. In the phase-integral method we must therefore avoid these points. Consequently, the solution to (1) is found by continuation on a path circumventing each transition point at a safe distance. This is done by following antiStokes separatrices toward and away from transition points and then jumping from one separatrix to another in the neighbourhood of a transition point, being careful to control the errors in so doing. It is instructive to look at exactly what happens along this path.

When we move on a path from one anti-Stokes separatrix to another, we cross a Stokes separatrix emanating from the same transition point, and the quantity $\left|\mathrm{e}^{\mathrm{i} w}\right|$ reaches an extremum there (see figure 1). When continuing the linear combination (11) across one of these Stokes separatrices the coefficient of the solution that is subdominant on that Stokes separatrix changes and the coefficient of the dominant solution remains roughly constant. This is the so-called Stokes phenomenon. In a series of recent papers, Berry $[38,39,40]$ has shown that this change enters smoothly. The change in the coefficient of the subdominant solution can qualitatively be compared to the error function. It grows rapidly in the vicinity of the Stokes separatrix and very slowly far away from it. The result is that, when we reach the second antiStokes separatrix, we have a different linear combination of the two solutions $f_{1}$ and $f_{2}$. Changes in the coefficients $a_{i}$ of this kind are necessary if we are to satisfy boundary conditions in both directions far from the transition point.

For our purposes, all we need is to find out how to continue a given linear combination from one anti-Stokes separatrix to another. The $F$-matrices do this for us. Below we give some $F$-matrix estimates that are useful in a phase-integral treatment of the quasinormal-mode problem. The situations analysed here are all illustrated in figure 1.

Let a collection of points $\left\{z_{i}, z_{i^{\prime}}\right\}$ lie sufficiently near a transition point that other transition points elsewhere in the complex plane do not affect the analysis. (If this condition cannot be satisfied, then one must perform an analysis for a so-cailed cluster of transition points.) Then there are a number of possible cases of interest.

- The points $z_{0}$ and $z_{1}$ lie on the same anti-Stokes line well away from a transition point. Then a linear combination of the two solutions is preserved to lowest order:

$$
F\left(z_{0}, z_{1}\right)=\left(\begin{array}{ll}
1 & 0 \\
0 & 1
\end{array}\right)+O(\mu) .
$$

- The points $z_{1}$ and $z_{2}$ lie on adjacent anti-Stokes separatrices emanating from a well isolated transition point. The curve joining them does not cross the cut emanating from the transition point, and where it crosses the Stokes separatrix emerging from the same point, the quantity $\left|\mathrm{e}^{\mathrm{i} w}\right|$ has a maximum. Then the linear combination changes as follows:

$$
F\left(z_{2}, z_{1}\right)=\left(\begin{array}{cc}
1 & 0 \\
-\mathrm{i} & 1
\end{array}\right)+\mathrm{O}(\mu)
$$

- The points $z_{3}$ and $z_{2}$ lie on adjacent anti-Stokes separatrices emanating from a well isolated transition point. The curve joining them does not cross the cut emanating 
from the transition point, and where it crosses the Stokes separatrix emerging from the same point, the quantity $\left|e^{i w}\right|$ has a minimum. Then we have

$$
F\left(z_{3}, z_{2}\right)=\left(\begin{array}{cc}
1 & -\mathrm{i} \\
0 & 1
\end{array}\right)+\mathrm{O}(\mu) .
$$

The formulae (26) and (27) are only valid when we have defined $w$ such that $w=0$ at the transition point.

Note that the two formulae can be remembered in a very simple way: from the knowledge that the coefficient of the solution that is subdominant on the Stokes separatrix will change, one can figure out which of the non-diagonal matrix elements is non-zero. Furthermore, this element is approximately $\pm \mathbf{i}$, with the sign depending on the direction (positive/negative) in which the transition point is circumvented.

- The point $z_{1}$ lies on an anti-Stokes separatrix and $z_{1}$ lies on a neighbouring anti-Stokes line that does not reach the transition point. The point $z_{1}$, is chosen in such a way that there is a Stokes line joining the two points. Suppose the quantity $\left|\mathrm{e}^{\mathrm{i} w}\right|$ increases monotonically as we move from $z_{1}$ to $z_{1^{\prime \prime}}$. Then we obtain from the basic estimates (23)

$F\left(z_{1}, z_{1}\right)=\left(\begin{array}{cc}1+\mathrm{O}(\mu) & \mathrm{O}(\mu) \\ \mathrm{O}(\mu)\left|\exp \left(2 \mathrm{i} \gamma_{11^{\prime}}\right)\right| & 1+\mathrm{O}(\mu)\left(1+\mathrm{O}(\mu)\left|\exp \left(2 \mathrm{i} \gamma_{11^{\prime}}\right)\right|\right)\end{array}\right)$

where we have defined

$$
\gamma_{11^{\prime}}=\int_{z_{1}}^{z_{1^{\prime}}} q \mathrm{~d} r
$$

and we have again set $w=0$ at the transition point. When the points $z_{1}$ and $z_{1}$, are very close to each other $\operatorname{Im} \gamma_{11^{\prime}}$ is necessarily small, and $F\left(z_{1^{\prime}}, z_{1}\right)$ is, to order $\mu$, a unit matrix. This important result means that we can 'jump' from one anti-Stokes line to a neighbouring one where convenient. It also will allow us in the next section to move the boundary condition at infinity from the real coordinate axis, where it is troublesome, to a suitable anti-Stokes line (separatrix), where it is well behaved.

It is now straightforward to show that the $F$-matrices connecting points lying on anti-Stokes lines that pass close to a well-isolated transition point are also given, to order $\mu$, by the expressions (26) or (27), as appropriate. For example, if the points $\left\{z_{i^{\prime}}\right\}$ lie on such lines, we find the following results.

- From (21) we obtain that the $F$-matrix connecting $z_{1^{\prime}}$ and $z_{2^{\prime}}$ across a Stokes separatrix on which $\left|\mathrm{e}^{\mathrm{i} w}\right|$ has a maximum is given by jumping from the first line to a nearby anti-Stokes separatrix that does go through the transition point, then connecting to the adjacent such line across the Stokes separatrix, and then jumping again to the desired anti-Stokes line. Algebraically, this means

$$
F\left(z_{2^{\prime}}, z_{1^{\prime}}\right)=F\left(z_{2^{\prime}}, z_{2}\right) F\left(z_{2}, z_{1}\right) F\left(z_{1}, z_{1^{\prime}}\right) \text {. }
$$

From (28) (and its inverse) we have that both $F\left(z_{2^{\prime}}, z_{2}\right)$ and $F\left(z_{1}, z_{1^{\prime}}\right)$ are identity matrices to order $\mu$. Therefore, we find that

$$
F\left(z_{2^{\prime}}, z_{1^{\prime}}\right)=\left(\begin{array}{cc}
1 & 0 \\
-\mathrm{i} & 1
\end{array}\right)+\mathrm{O}(\mu)
$$


- Analogously, we can show that the $F$-matrix connecting $z_{3^{\prime}}$ and $z_{2^{\prime \prime}}$ across a Stokes separatrix on which $\left\{e^{i w}\right\}$ has a minimum is given by

$$
F\left(z_{3^{\prime}}, z_{2^{\prime \prime}}\right)=\left(\begin{array}{cc}
1 & -i \\
0 & 1
\end{array}\right)+\mathrm{O}(\mu) .
$$

It should be noted that in a situation where several transition points are close to each other the elements of the $F$-matrices are not independent of the order of approximation used. General formulae relevant for studying clusters of transition points are discussed by Fröman et al in [30]. However, although their formulae may be used in some situations (see for example [15]) they cannot be used to determine explicit expressions for the $F$-matrix elements. Such expressions can be obtained by using uniform approximations (that are uniformly valid in all regions of the coordinate-plane) [31]. In order to do this a comparison with an exactly soluble equation is made. The exact solution is then locally mapped onto the problem under consideration. Basically only three such exact solutions are known: the Airy functions (one transition point), the parabolic cylinder (Weber) functions (two transition points) and the Coulomb wavefunctions (two transition points and a second-order pole). These last two cases were used by Andersson and Linnæus in an analysis of the black-hole problem [19]. However, Araújo et al [36] have shown that the case of an isolated cluster of two transition points can be treated using formulae very similar to the ones described in this paper.

From the discussion in this section, it should now be clear that, as with most asymptotic approximation methods, its application is as much an art as a science. The choice of $Q$ is delicate, and a suitable function is not guaranteed. One has to ensure that the errors, which rise steeply near the transition points, are small elsewhere; in particular, one wants a region near a transition point where one can jump from one anti-Stokes separatrix to another without significant error. In our view, the study of errors in the phase-integral method is not yet sufficiently well developed, and we will come back to this point below.

\section{Phase-integral analysis of the black-hole problem}

In order to treat the full black-hole problem, we must add to the transition-point analysis a treatment of the boundary conditions both far from the hole and at the horizon. We consider the horizon first.

\subsection{Treatment of singularity at the horizon.}

In the case of a Schwarzschild black hole, the function $R_{\ell}(r)$, as given by (3) and (4), is singular both at the origin and at the event horizon. The latter singularity is cause for concern since it is at the horizon that one should place a boundary condition. From (3) and (4) we have that

$$
\lim _{r \rightarrow 2}(r-2)^{2} R_{\ell}(r)=4 \sigma^{2}+\frac{1}{4} .
$$

If we define the constant $\alpha$ by

$$
\alpha(\alpha+1) \equiv-\left[4 \sigma^{2}+\frac{1}{4}\right]
$$


then the elementary theory of differential equations shows that near the horizon (a second-order pole of $R_{\ell}$ )

$$
\Psi \sim\left\{\begin{array}{l}
(r-2)^{\alpha+1} \\
(r-2)^{-\alpha}
\end{array} \quad \text { as } r \rightarrow 2 .\right.
$$

The WKB approximation, on the other hand, using $q=R_{\ell}^{1 / 2}$, generates approximate solutions with the behaviour

$$
f_{1,2}^{\mathrm{WKB}}(r) \sim(r-2)^{(1 / 2) \mp \sqrt{\alpha(\alpha+1)}}
$$

as $r \rightarrow 2$. This is unsatisfactory, and tells us that we should look for a better first guess for $Q$ than $R_{\ell}^{1 / 2}$. It does not take long to see that if one chooses [15]

$$
Q^{2}(r)=R_{\ell}(r)-\frac{1}{4(r-2)^{2}}
$$

then one has

$$
\lim _{r \rightarrow \infty} Q^{2}(r)=\sigma^{2}
$$

and

$$
\lim _{r \rightarrow 2}(r-2)^{2} Q^{2}(r)=4 \sigma^{2}
$$

which leads to the same behaviour near the horizon as the exact solutionequation (35). With this choice of $Q^{2}$, there are four transition points. For the slowest damped modes, they remain well separated, and the analysis of the previous section applies to each of them.

Interestingly, there is another way to justify this function $Q^{2}$ : if we were to take $Q^{2}=R_{\ell}$, then we would find that $\epsilon_{0}$ remains finite at the horizon. For the choice (37), the error term $\epsilon_{0}$ goes to zero there. Hence, the two solutions (12) tend to exactness as the horizon is approached.

\subsection{Global structure and asymptotic behaviour of solutions}

Because we need the function $Q(r)$, not $Q^{2}(r)$, we must introduce cuts in the complex plane at every transition point. The necessary cuts can always be placed in a way such that they need not be crossed in the continuation of our approximate solutions to (1). There is, of course, no unique way of cutting the complex plane, but our analysis would become very abstract and difficult to follow if we were to proceed without defining where cuts lie at this point. Nevertheless, there is no loss of generality in the following presentation.

From now on we assume that the necessary cuts are introduced as in figure 2. We choose the overall phase of $Q$ such that

$$
\lim _{r \rightarrow \infty} Q(r)=\sigma
$$




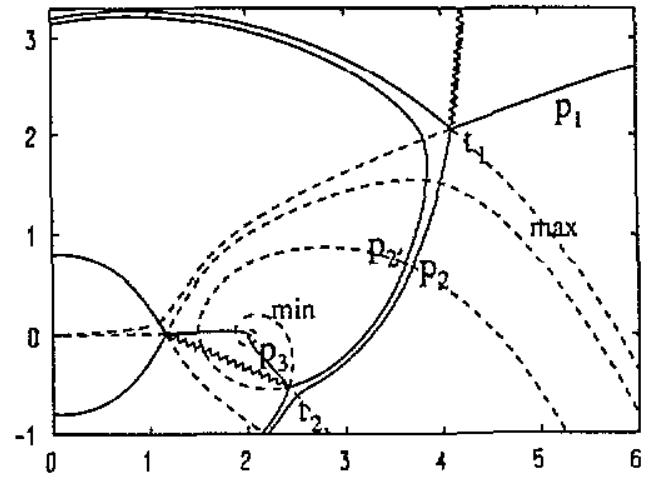

Figure 2. Pattern of Stokes (broken) and anti-Stokes (full) separatrices emanating from the transition points for the fundamental quasinormal mode ( $\sigma=0.3737-0.0889 i$ ). Cuts are represented by the zigzagged lines. The labels max and min on the Stokes separatrices denote maxima and minima of the function $|\exp (\mathrm{i} w)|$ respectively. Three of the four transition points lie in the region of the plot. The ones we use for the analysis are labelled $t_{1}$ and $t_{2}$. The third, unlabelled, is near $r=1$. The cut from $t_{1}$ terminates on the unseen fourth transition point, so that $Q$ is single-valued at infinity.

(Note that this is well defined, since the cuts are taken to leave $Q$ single-valued at infinity.) Having chosen the phase of $Q$ in this way, the disposition of the cuts determines the phase at all other points. The cuts introduced in figure 2 lead to

$$
\lim _{r \rightarrow 2}(r-2) Q(r)=2 \sigma .
$$

Note that this does not correspond to the situation investigated by Araújo et al [36] or Froman et al [15], since they introduced the cuts in a different way. In two recent investigations by Andersson and Linnæus [19] and by the present authors [16] the cuts are introduced in the way described above.

From (40) and (41) and our choice of time factor, we can identify $f_{1}(r)$, as defined in (12), with the desired outgoing-wave solution as $r \rightarrow \infty$, and $f_{2}(r)$ with the waves falling across the horizon as $r \rightarrow+2$. It follows that a quasinormal-mode solution $\Psi$ is defined by the asymptotic behaviour

$$
\Psi \underset{r \rightarrow+2}{\sim} a_{2} f_{2}(r)
$$

and

$$
\Psi \underset{r \rightarrow+\infty}{\sim} a_{1} f_{1}(r)
$$

where $a_{2} \equiv a_{2}(2)$ and $a_{1} \equiv a_{1}(\infty)$ are undetermined normalization factors. Again, we stress that the solutions are single-valued at infinity.

\subsection{Boundary conditions on anti-Stokes separatrices}

The introduction of these boundary conditions on the real coordinate axis leads to well known numerical difficulties [25,14]. Given our choice of time factor, the desired solutions (42) and (43) increase exponentially as we approach the event horizon and 
infinity along the real axis, and the solutions that do not fit the boundary condition decrease exponentially. In order to determine the frequency of a quasinormal mode, we have to find the exponentially decreasing term to decide whether, for any given frequency, it is or is not present. Given the finite accuracy of any numerical scheme, this is a delicate problem, since at some critical value $r_{\mathrm{c}}$ of the coordinate, the decreasing term will become smaller than the error in the increasing one.

To avoid this difficulty, we note that the asymptotic conditions (42) and (43) can be applied on any line approaching the horizon and infinity, respectively. In particular, the boundary conditions can be introduced on anti-Stokes separatrices (see $[36,15,21]$ ), where the two solutions (12) are essentially of the same order of magnitude, and the undesired solution can easily be singled out. In this paper we will implement this idea analytically, to get approximations to the quasinormal modes. But we note that the same thing can be done numerically, albeit at the cost of finding the appropriate anti-Stokes lines numerically. This would be a powerful way to find numerical values for the quasinormal mode frequencies with an accuracy limited only by machine arithmetic precision [21]. In cases where the full analytic treatment of the present paper is not available, such as for modes of stars whose structure is known only from numerical integrations and therefore cannot be extended into the complex plane, the troublesome boundary condition at infinity can still be handled this way. We intend to treat this case in a future paper.

We consider first the condition at infinity. We would like to introduce the condition (43) on the anti-Stokes separatrix emanating from the transition point $t_{1}$ and running towards infinity (see figure 2). Let us consider a point situated on this anti-Stokes separatrix and a point on the real axis. It has been shown by Araújo et al [36] that there exist paths such that the $\mu$-integral between these points tends to zero as the points tend to infinity. This $\mu$-integral provides an error bound, and the solution (43) is exponentially increasing if continued from the anti-Stokes separatrix to the real axis. Hence, it follows from the basic estimates (23) that the F-matrix connecting these points is exactly the identity matrix. The boundary condition, as imposed on the anti-Stokes separatrix, therefore implements the correct condition on the real axis. Alternatively it can be argued that, since $\epsilon \rightarrow 0$ as we approach infinity, the single-valuedness of $Q$ (and therefore $w$ ) means that we can use the asymptotic form $\exp (i w)$ anywhere near infinity. Hence, once the desired sign of the exponent has been identified we can automatically use it on the anti-Stokes separatrix running in the same direction.

We will also introduce the condition (42) on the anti-Stokes separatrix extending from the transition point $t_{2}$ towards the horizon (see figure 2). In the appendix we demonstrate that our choice of $Q^{2}(r)$ and the boundary condition for quasinormal modes at the horizon allow us to write

$$
a_{1}(r)=F_{12}(r,+2) a_{2}(+2) \quad a_{2}(r)=F_{22}(r,+2) a_{2}(+2) .
$$

These equations have the same significance for the boundary condition at the horizon as we have just found for the one at infinity. One can apply this boundary condition on an anti-Stokes separatrix and follow that curve out to a transition point. This can be done either analytically (as we do below) or fully numerically.

\subsection{Example: a simple derivation of the Bohr-Sommerfeld formula}

As an example of the application of the phase-integral method to the determination of the characteristic frequencies of quasinormal modes, we derive a formula for 
the Schwarzschild case. The formula is valid under the simplifying assumption that only two well separated transition points are relevant for our analysis. This will be applicable to the first few modes in the infinite sequence, and corresponds to the situation depicted in figure 2 .

As mentioned in the previous section the boundary condition of outgoing waves at infinity is imposed on the anti-Stokes separatrix that emerges from the transition point $t_{1}$ towards infinity. The $F$-matrix connecting two different points on this antiStokes line, both lying far away from $t_{1}$, is approximately a unit matrix. Hence, using (21), the solution (43) can be extended from infinity to the point $p_{1}$ (situated sufficiently far away from the transition point for the integral $\mu\left(p_{1}, \infty\right)$ to be small), and we obtain

$$
\Psi\left(p_{1}\right)=a_{1}(\infty) f_{1}\left(p_{1}\right) .
$$

In order to continue this solution to the point $p_{2}$, the transition point $t_{1}$ must be circumvented at a safe distance, that is, one must choose a path connecting $p_{1}$ to $p_{2}$ such that $\mu\left(p_{2}, p_{1}\right)$ is small. In doing this, a single maximum of $\left|\mathrm{e}^{\mathrm{j} w}\right|$ will be crossed. It follows from a comparison with figure 1 that the $F$-matrix relating the solution at these two points is given by (26). Therefore, from (45) and (21) we find that

$$
\Psi\left(p_{2}\right)=a_{1}(\infty)\left[f_{1}\left(p_{2}\right)-\mathbf{i} f_{2}\left(p_{2}\right)\right] .
$$

So far the transition point $t_{1}$ has been used as phase-reference level for the functions $f_{i}$, i.e. it is the lower limit of the integral in (6) in the first-order approximation or the encircled point in the calculation of (24) in higher orders of approximation. We could carry on doing so, but it will simplify the matching of solutions to be made later, if we use $t_{2}$ as reference level. That means we work with a different definition $\bar{f}_{1,2}$ of the fundamental solutions, differing by a constant phase:

$$
\tilde{f}_{1}=\mathrm{e}^{\mathrm{i} \gamma_{21}} f_{1} \quad \tilde{f}_{2}=\mathrm{e}^{-\mathrm{i} \gamma_{21}} f_{2}
$$

where, in the first order of approximation,

$$
\gamma_{21}=\int_{t_{2}}^{t_{1}} q \mathrm{~d} r .
$$

In higher orders of approximation this definition must be replaced by the contour integral, cf (24),

$$
\gamma_{21}=\frac{1}{2} \oint_{\Gamma_{21}} q \mathrm{~d} r
$$

where the contour $\Gamma_{21}$ is to encircle the two transition points $t_{1}$ and $t_{2}$. Now (46) takes the form

$$
\Psi\left(p_{2}\right)=a_{1}(\infty)\left[\mathrm{e}^{-\mathrm{i} \gamma_{21}} \vec{f}_{1}\left(p_{2}\right)-\mathrm{ie}^{\mathrm{i} \gamma_{21}} \bar{f}_{2}\left(p_{2}\right)\right] .
$$

We now consider the boundary condition of purely ingoing waves falling across the event horizon (42) that is imposed on the anti-Stokes separatrix that emanates from $t_{2}$ towards the horizon. Since the $F$-matrix connecting two points on the same 
anti-Stokes line is asymptotically a unit matrix, we immediately obtain from (44) (provided that $\mu\left(p_{3}, t_{2}\right)$ is small) that the solution at point $p_{3}$ is

$$
\Psi\left(p_{3}\right)=a_{2}(2) \tilde{f}_{2}\left(p_{3}\right)
$$

We want to continue this solution to the point $p_{2}$, near $p_{2}$. We must then circumvent the transition point $t_{2}$ along a path such that $\mu\left(p_{2^{\prime}}, p_{3}\right)$ is small. In doing this, a single minimum of $\left|\mathrm{e}^{\mathrm{i} w}\right|$ is crossed. Hence, the $F$-matrix connecting $p_{2}$, with $p_{3}$ is given by (27), cf figure 1 . We obtain

$$
\Psi\left(p_{2^{\prime}}\right)=a_{2}(2)\left[-\mathbf{i} \tilde{f}_{1}\left(p_{2^{\prime}}\right)+\tilde{f}_{2}\left(p_{2^{\prime}}\right)\right] .
$$

Finally, we want to continue the solution to $p_{2}$. We take a Stokes line as the path connecting $p_{2}$ to $p_{2}$. Using the basic estimates (23) we find that

$$
F\left(p_{2}, p_{2^{\prime}}\right)=\left(\begin{array}{cc}
1+\mathrm{O}(\mu) & \mathrm{O}(\mu) \\
\mathrm{O}(\mu)\left|\exp \left(2 \mathrm{i} \gamma_{21}\right)\right| & 1+\mathrm{O}(\mu)\left(1+\mathrm{O}(\mu)\left|\exp \left(2 \mathrm{i} \gamma_{21}\right)\right|\right)
\end{array}\right) .
$$

We have used the fact that $\operatorname{Im} \gamma_{22^{\prime}}=\operatorname{Im} \gamma_{21}$. Provided that $\left|\exp \left(i \gamma_{21}\right)\right| \sim 1$ we can approximate $F\left(p_{2}, p_{2^{\prime}}\right)$ by the identity matrix (to order $\mu$ ) and obtain

$$
\Psi\left(p_{2}\right)=a_{2}(2)\left[-\mathrm{i} \tilde{f}_{1}\left(p_{2}\right)+\tilde{f}_{2}\left(p_{2}\right)\right] .
$$

The two expressions (50) and (54) must be identical within the approximation. Hence, we must have

$$
a_{1}(\infty) \exp \left(-\mathrm{i} \gamma_{21}\right)=-\mathrm{i} a_{2}(2) \quad-\mathrm{i} a_{1}(\infty) \exp \left(\mathrm{i} \gamma_{21}\right)=a_{2}(2)
$$

Dividing these two equations yields the phase-integral condition determining a quasinormal-mode solution

$$
\exp \left(2 \mathrm{i} \gamma_{21}\right)=-1
$$

Taking the logarithm of this expression we obtain the Bohr-Sommerfeld formula

$$
\gamma_{21}=\left(n+\frac{1}{2}\right) \pi
$$

It follows from our choice of phase for $Q$ that $\operatorname{Re} \gamma_{21}$ must always be positive, and $n$ is therefore a non-negative integer labelling the modes.

In the analysis leading to (57) we assumed that the two transition points considered, $t_{1}$ and $t_{2}$, were well separated from each other as well as from all other transition points. This is, in fact, not a necessary requirement for the validity of the formula. Alternative analyses by Araújo et al [36] and Fröman et al [15], yield that the formula remains valid even if the two transition points considered lie close together. Nevertheless, all other transition points must be remote.

Numerical calculations using the WKB Bohr-Sommerfeld formula (which is (57) with $q$ in the integrand (48) for $\gamma_{21}$ replaced by $R_{\ell}^{1 / 2}$ ) were performed by Guinn $e t$ al [14] and with the present Bohr-Sommerfeld formula by Fröman et al [15]. The formula is also discussed in a further paper by the present authors [16]. It is very 
accurate for quasinormal modes such that $|\operatorname{Re} \sigma|>|\operatorname{Im} \sigma|$, but not reliable when $\operatorname{Im} \sigma \mid$ increases. Hence, for the Schwarzschild black hole it can be used to determine the lowest-lying modes. In a real astrophysical situation, it is probable that only the first few modes will be extracted from the gravitational waveform. The simple derivation above (or the more general analysis in [36] and [15]) leads to a condition that accurately determines those quasinormal-mode frequencies that are likely to be of the most physical interest.

Our assertions about the accuracy of the Bohr-Sommerfeld formula are based on comparing its results with the accurate numerical calculations of Leaver [1], which have been subsequently verified by others [21,12]. Interestingly, if we ask for the intrinsic error test, namely the values of $\mu$, they are rather large: in our experience it is in fact hard to find trajectories that make $\mu$ very small. Given the success of the phase-integral formulae, we have come to the conclusion that bounds obtained using $\mu$ overestimate in a serious way the actual errors in the calculation.

It is not hard to see how this might happen. The quantity $\mu$ in fact often arises as a bound on integrals of the type (cf (7) and (10))

$$
\int \epsilon \phi_{ \pm} \mathrm{d} w \sim \int \epsilon \mathrm{e}^{ \pm \mathrm{i} w} \mathrm{~d} w
$$

which are bounded by $\int|\epsilon \mathrm{d} w|$, but which may in fact be much smaller than this bound because of the cancellations inside the integration region produced by the oscillations of the exponential $\phi_{ \pm}$. Better limits on the errors can probably be obtained by integrating such expressions by parts and looking at bounds on $\mathrm{d} \epsilon / \mathrm{d} w$ : if the function $\epsilon$ is smooth, then the integrals above should be small even if $\epsilon$ is not always very small itself.

This is one area of this subject that needs further study: a more accurate bound on the errors would be very helpful when the method is applied to problems for which calculations by other methods are not available.

\section{Acknowledgments}

NA thanks the Royal Society and The Royal Swedish Academy of Sciences for financial support. MEA wishes to thank Coordenaçāo de Aperfeiçoamento de Pessoal de Ensino Superior (CAPES) and the Royal Society for financial support during different periods while this work was being carried out.

\section{Appendix}

The purpose of this appendix is to prove the two formulae

$$
a_{1}(r)=F_{12}(r,+2) a_{2}(+2)
$$

and

$$
a_{2}(r)=F_{22}(r,+2) a_{2}(+2)
$$

with mathematical rigour. Although (21), defining the $F$-matrix, together with the boundary condition of no outgoing waves at the event horizon make them seem 
plausible it is necessary to be careful. Namely, the formulae represent limiting values that must exist and be finite if they are to make sense.

We have already seen that our choice of $Q^{2}(37)$ guarantees that the $\mu$-integral tends to a finite limit as $r$ tends to 2 (and that $\epsilon \rightarrow 0$ ). The phase-integral method then gives satisfactory results in the neighbourhood of $r=2$ since our approximate solutions tend to exactness.

Let $r_{0}$ be a point in the complex plane such that $a_{1}\left(r_{0}\right)$ and $a_{2}\left(r_{0}\right)$ are finite, and $\Lambda$ be a path connecting $r_{0}$ to the horizon, along which $|\exp [-i w(r)]|$ is monotonically increasing and the $\mu$-integral convergent. It can be shown, in analogy with a demonstration given in [26], that under these circumstances the two formulae

$$
F_{11}\left(r_{0},+2\right) \equiv \lim _{r \rightarrow+2} F_{11}\left(r_{0}, r\right) \text { exists and is finite }
$$

and

$$
F_{21}\left(r_{0},+2\right) \equiv \lim _{r \rightarrow+2} F_{21}\left(r_{v}, r\right) \quad \text { exists and is finite }
$$

are valid (with the limits calculated along $\Lambda$ ). These formulae form the basis of the analysis below.

By letting $r$ tend to +2 in the $i=2$ component of (21) we immediately obtain

$a_{2}(+2) \equiv \lim _{r \rightarrow+2} a_{2}(r)=F_{21}\left(+2, r_{0}\right) a_{1}\left(r_{0}\right)+F_{22}\left(+2, r_{0}\right) a_{2}\left(r_{0}\right)$

From (A3), (A4) and the inverse of the matrix $F\left(r_{0},+2\right)$, it follows that $F_{21}\left(+2, r_{0}\right)$ and $F_{22}\left(+2, r_{0}\right)$ in (A5) exist and are finite, which in turn implies that $a_{2}(+2)$ exists and is finite.

Now, the $i=1$ component of (21) gives

$$
a_{1}(r)=F_{11}\left(r, r_{0}\right) a_{1}\left(r_{0}\right)+F_{12}\left(r, r_{0}\right) a_{2}\left(r_{0}\right)
$$

Using (A5) to replace $a_{2}\left(r_{0}\right)$ in this expression and taking the limit $r \rightarrow+2$ we obtain

$$
a_{1}(+2) \equiv \lim _{r \rightarrow+2} a_{1}(r)=\frac{a_{1}\left(r_{0}\right)}{F_{11}\left(r_{0},+2\right)}+\frac{F_{12}\left(+2, r_{0}\right) a_{2}(+2)}{F_{11}\left(r_{0},+2\right)} .
$$

The boundary condition of no waves coming out of the event horizon leads to the vanishing of this limit. Since $F_{11}\left(r_{0},+2\right)$ is finite, this establishes the first of our results, equation (A1).

Similarly, the $i=2$ component of $(21)$ is

$$
a_{2}(r)=F_{21}\left(r, r_{0}\right) a_{1}\left(r_{0}\right)+F_{22}\left(r, r_{0}\right) a_{2}\left(r_{0}\right)
$$

Replacing $a_{2}\left(r_{0}\right)$ according to (A5) and using the multiplication rule for $F$-matrices we obtain

$$
a_{2}(r)=\frac{F_{21}(r,+2) a_{1}\left(r_{0}\right)}{F_{11}\left(r_{0},+2\right)}+\frac{F_{22}\left(r, r_{0}\right) a_{2}(+2)}{F_{11}\left(r_{0},+2\right)}
$$

Using (A1) to replace $a_{1}\left(r_{0}\right)$ the second result, (A2), follows after a little algebra. 


\section{References}

[1] Leaver E W 1985 An analytic representation for the quasinormal modes of Kerr black holes Proc. R. Soc. A 402 285-98

[2] Kokkotas K D and Schutz B F 1986 Normal modes of a model radiating system Ger. Rel. Grav 18 913-21

[3] Kokkotas K D and Schutz B F 1992 W-modes: a new family of normal modes of pulsating relativistic stars Mon. Not. R. Astron Soc. 255 119-28

[4] Kojima Y 19882 families of normal-modes in relativistic stars Prog. Theor. Phys. 79 665-75

[5] Chandrasekhar S 1983 The Mathematical Theory of Black Holes (New York: Oxford University Press)

[6] Piran T and Stark R F 1986 Numerical relativity, rotating gravitational collapse and gravitational radiation Dynamical Spacetimes and Numerical Relativity ed J M Centrella (Cambridge: Cambridge University Press) pp 40-73

[7] Sun Y and Price R H 1988 Excitation of quasinormal ringing of a Schwarzschild black hole Phys. Rev. D 38 1040-52

[8] Leaver E W 1986 Spectral decomposition of the perturbation response of the Schwarzchild geometry Phys. Rev. D 34 384-408

[9] Abramovici A et al 1992 LIGO: the laser interferometer gravitational-wave observatory Science 256 325-33

[10] Bradaschia C et al 1990 The Virgo project-a wide band antenna for gravitational-wave detection Nuch Instrum. Methods Phys. A 518-25

[11] Friedman J L 1986 Stability of relativistic stars and black holes Highlights in Gravitation and Cosmology ed B R lyer, A Kembhavi, J V Narlikar and C V Vishveshwara (Cambridge: Cambridge University Press) pp 361-74

[12] Nollert H-P and Schmidt B G 1992 Quasinormal modes of Schwarzschild black holes: defined and calculated via Laplace transformation Phys. Rev. D 45 2617-27

[13] Ferrari V and Mashhoon B 1984 New approach to the quasinormal modes of a black hole Phys. Rev. D 30 295-304

[14] Guinn J W, Will C M, Kojima Y and Schutz B F 1990 High-overtone normal modes of Schwarzschild black holes Class. Quantum Grav. 7 LA7-LS3

[15] Fröman P O, Fröman N, Andersson $N$ and Hökback A 1992 Black-hole normal modes: phase-integral treatment Phys. Rex. D 45 2609-16

[16] Andersson N, Araújo M E and Schutz B F 1993 Generalized Bohr-Sommerfeld formula for black hole normal modes Class. Quantum Grav, $10757-65$

[17] Nollert H-P 1986 Über Quasi-Normalmoden Schwarzschildscher Schwarzer Löcher Diplom thesis University of Köln

[18] Nollert H-P 1992 The Sixth Marcel Grassman Meeting on General Relativity ed H Sato (Singapore: World Scientiffc) in press

[19] Andersson N and Linnæus S 1992 Quasinormal modes of a Schwarzschild black hole: Improved phase-integral treatment Phys. Rev: D 46 4179-87

[20] Andersson $\mathrm{N} 1992$ On the asymptotic distribution of quasinormal-mode frequencies for Schwarzschild black holes Class. Quantum Grav. Submitted

[21] Andersson N 1992 Accurate investigation of black-hole normal modes Proc. $R$ Soc. A 439 47-58

[22] Regge T and Wheeler J A 1957 Stability of a Schwarzschild singularity Phys. Rev. 108 1063-9

[23] Zerilli F J 1970 Gravitational field of a particle falling in a Schwarzschild geometry analyzed in tensor harmonics Phys. Rev. D 10 2141-60

[24] Chandrasekhar S 1975 On the equations governing the perturbations of the Schwarzschild black hole Proc. $R$. Soc. A 343 289-98

[2S] Chandrasekhar S and Detweiler S 1975 The quasi-normal modes of the Schwarzschild black hole Proc. $R$ Sac. A $344441-52$

[26] Fröman P O and Fröman N 1965 swKB Approximation, Contributions to the Theory (Amsterdam: North-Holland)

[27] Fröman N 1966 Outline of a general theory for higher-order approximations of the JWKB-type Ank. Fys. 32 541-8

[28] Fröman N and Fröman P O 1974 A direct method for modifying certain phase-integral approximations of arbitrary order Ann. Phys. 83 103-7 
[29] Fröman N and Fröman P O 1992 Phase-integral approximation of arbitrary order generated from an unspecified base function Forty More Years of Ramifications: Spectral Asymptotics and its Applications (Discourses in Mathematics and its Applications, no 1) ed S A Fulling and F J Narcowich (Texas A\&M University, Department of Mathematics) pp 121-59

[30] Fröman N, Fröman P O and Lundborg B 1988 The Stokes constants for a cluster of transition points Math Froc. Camb. Phil. Soc. 104 153-79

[31] Berry M V and Mount K E 1972 Semiclassical approximations in wave mechanics Rep. Prog. Phys. 35 315-97

[32] Heading I 1962 An Introduction to Phase-integral Methods (London: Methuen)

[33] Mathews J and Walker R L 1970 Mathematical Methods of Physics 2nd edn (Cambridge, MA: Addison-Wesley)

[34] Bender C M and Orszag S A 1978 Advanced Mathematical Methods for Scientists and Engineers (New York: McGraw-Hill)

[35] Dammert Ö 1983 Transmission through a system of potential barriers. I. Transmission coefficient $J$. Math. Phys. 24 2163-75

[36] Araújo M E, Nicholson D and Schutz B F 1993 Class. Quantum Grav submitted

[37] Fröman N 1970 Connection formulas for certain higher order phase-integral approximations Ann. Phys. 61 451-64

[38] Berry M V 1989 Uniform asymptotic smoothing of Stokes's discontinuities Proc. $R$. Soc. A 422 7-21

[39] Berry M V 1989 'Stokes' Phenomenon; smoothing a Victorian discontinuity Publ Math Institut des Hautes Etudes Scientifique 68 211-21

[40] Berry M V 1990 Waves near Stokes lines Proc. $R$. Soc. A 427 265-80 